\title{
Electrical potential difference of colonic mucosa
}

\author{
C. J. EDMONDS ${ }^{1}$
}

From the MRC Department of Clinical Research, University College Hospital Medical School, London

Epithelia which separate disparate media are polarized and a frequently observed aspect of this polarization is an electrical potential difference (pd) between the two epithelial faces. The epithelium of gut, whose cells on one side are bathed by an almost constant medium, the extracellular fluid, and on the other face by a luminal medium of very variable composition, has a fairly stable pd across it, the serosal or blood side being generally positively charged with respect to the lumen. The pd arises in the epithelial layer remaining unchanged when the muscle coats are removed. It is referred to as the transmucosal pd and the present account will be concerned with describing some of its characteristics.

Its magnitude varies in different parts of the intestine. The pd is usually less than $10 \mathrm{mV}$ in the jejunum and ileum but rises when certain nonelectrolytes, such as glucose and amino acids, are added to the luminal solution (Barry, 1967). In the colon, the pds are higher, in normal man ranging from $30-40 \mathrm{mV}$, while in healthy individuals on a normal diet there is relatively little variation along the length of the organ (Geall, Code, McIlrath, and Summerskill, 1969). A further contrast to small intestine is that the pd is unaffected by glucose and amino acids in the lumen.

Measurement of the transmucosal pd depends on being able to place an electrode on either side of the epithelium and on having a millivoltmeter with a high input impedance. The latter characteristic is necessary as the electrodes and solutions have a relatively high electrical resistance and it is important that the meter should draw little current. The electrodes incorporate a reversible junction electrode-either a calomel cell or $\mathrm{Ag}-\mathrm{AgCl}$ junctionin order to provide a stable connexion between the solutions and leads. An example of such a system for measurement of pds of rectum and sigmoid colon under direct vision is shown in figure 1 . It is not ordinarily practicable in human studies to place an electrode directly on the submucosal side of the epithelium, and, since normal skin has a significant and variable electrical charge of its own, the electrode

${ }^{1}$ Address for correspondence: Radioisotope Division, Clinical Research Centre, Northwick Park Hospital, Watford Road, Harrow, Middx. HA1 3UJ.
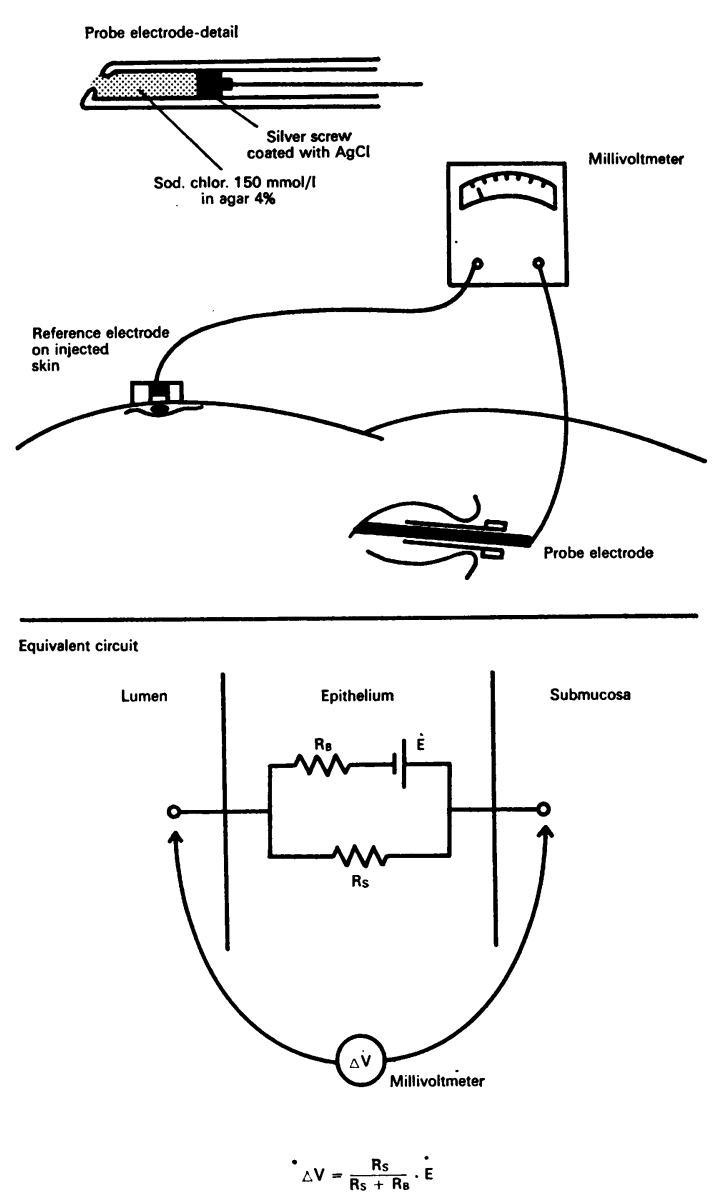

Fig 1 Diagrammatic representation of method of measurement of transmucosal pd in the rectum and distal colon. Both probe and reference electrodes incorporate silver-silver chloride junctions shown in detail for the probe. The equivalent circuit is also illustrated. $E, \Delta V, R_{b}$ and $R_{s}$ represent the $E M F$ generated by the active transport mechanisms, the measured transmucosal pd, and the internal resistance and shunt resistance respectively. 
cannot be satisfactorily placed on the unprepared skin surface (Anderssen and Grossman, 1965). Subcutaneous and intravenous electrodes overcome the problem but are inconvenient in practice. Abrasion of the skin is both unpleasant and unreliable (Grantham, Code, and Schlegel, 1970). An easier satisfactory method is an intradermal saline injection which abolishes the skin pd for at least 30 minutes (Archampong and Edmonds, 1972). Fortunately there is no other significant source of EMF between the subcutaneous and submucosal tissues, so that this method allows the transmucosal pd to be measured as a simple and rapid clinical procedure.

When a pd is recorded across an epithelium, some thought has to be given to its origin. A number of possibilities must be considered. If there exists a difference of composition between the solutions bathing the two sides of the epithelium, then significant pds (osmotically induced and diffusion potentials) can arise simply from this difference. Such pds can be of considerable biological interest and importance but they need to be differentiated from the transfer pds generated by the epithelial cells themselves and which exist in the absence of transmucosal concentration gradients. The pds due to osmotic gradients (sometimes called 'streaming potentials') arise largely from the flow of fluid, due to osmotic pressure gradients through charged pores. Osmotically induced pds have been demonstrated in the gallbladder and small intestine (Diamond and Harrison, 1966; Smyth and Wright, 1966) but not in the colon. Much more important for colonic studies are pds due to transmucosal difference in ionic concentration. These diffusion potentials result from the different mobility of various ions when moving through solutions or tissues. Consequently, if the solution on the luminal side differs much from that on the serosal side of the epithelium, a considerable transmucosal pd on that account alone may be recorded.

The transfer pd is associated with the active transport of charged particles from one side of the epithelium to the other, and is coupled to metabolic processes so that the transfer pd depends on the integrity of the cellular mechanisms. Thus when colonic epithelium is examined in vitro, it can be shown that anoxia, metabolic inhibitors, like dinitrophenol, and cardiac glycosides, such as ouabain, will reduce or abolish the pd and eliminate active transport (Edmonds and Marriott, 1968). The transfer pd is also influenced by the amount of substance to be transported. In the case of the colon, sodium is the ion which appears to be actively transported in the greatest quantity but lowering the luminal sodium concentration does not alter the $\mathrm{pd}$ by very much (Dalmark, 1970; Archampong and
Edmonds, 1972). This is probably because such a change also involves altering ionic gradients in the system so introducing diffusion potentials and changes in electrical resistance which mask effects on the transfer potential difference.

A very simple electrical model or equivalent circuit is useful in describing some properties of the pd (fig 1). From such a model, it is evident that the measured pd $(\Delta \mathrm{V})$ is obviously less than transport generated EMF (E) because of the presence of a shunt $\left(R_{s}\right)$ associated with the passive movement of ions through the epithelium. Furthermore the model shows that a change in the measured pd may be due either to an alteration in the activity of the ion pump (E) or to a change in the shunt resistance $\left(R_{\mathbf{s}}\right)$. If, for example, the tissue becomes more 'leaky' the freer movement of ions leads to a reduction in $\mathbf{R}_{\mathrm{s}}$ and hence $\Delta \mathrm{V}$ although $\mathrm{E}$ may be unchanged. The simple electrical model is usually satisfactory for considering the electrical properties shown by transmucosal measurements when the epithelium is treated as a 'black box'. It proves inadequate, however, for understanding the pd profiles within the colonic epithelium as revealed by microelectrode studies (Edmonds and Nielsen, 1968). To represent electrically these results, more complex equivalent circuits are needed (Schultz, 1972). Here we are restricted to the discussion of transmucosal phenomena.

The pure equivalent circuit should demonstrate all the electrical characteristics of the epithelium but gives no information about the ionic fluxes and so a chemical model is also needed. The electrical and chemical models are of course related to each other (Finkelstein and Mauro, 1963). A complete chemical model would describe the system in terms of the chemical concentrations in the various compartments -lumen, intracellular and extracellular - and would describe the transmembrane potentials, the ionic pumps and fluxes, and their characteristics. We are far from this but as sodium is the major ion actively transported, sodium absorption taking place in large quantity against the electrochemical gradient and, as the net sodium flux is closely correlated with short circuit current in human colon studied in vitro (Archampong, Harris, and Clark, 1972), it seems very likely that active sodium absorption is the major factor in the transfer pd of colonic mucosa. Chloride normally provides the main ion for carrying current in the shunt $\left(\mathbf{R}_{\mathbf{s}}\right)$. Consistent with this view too is the effect of substituting other anions for chloride, a rise of pd occurring with increasing anion size and hence increasing value of $R_{s}$ (Archampong and Edmonds, 1972).

The transmucosal colonic pd can be considerably influenced by some hormones and drugs. The mineralocorticoids are especially potent and the pd 


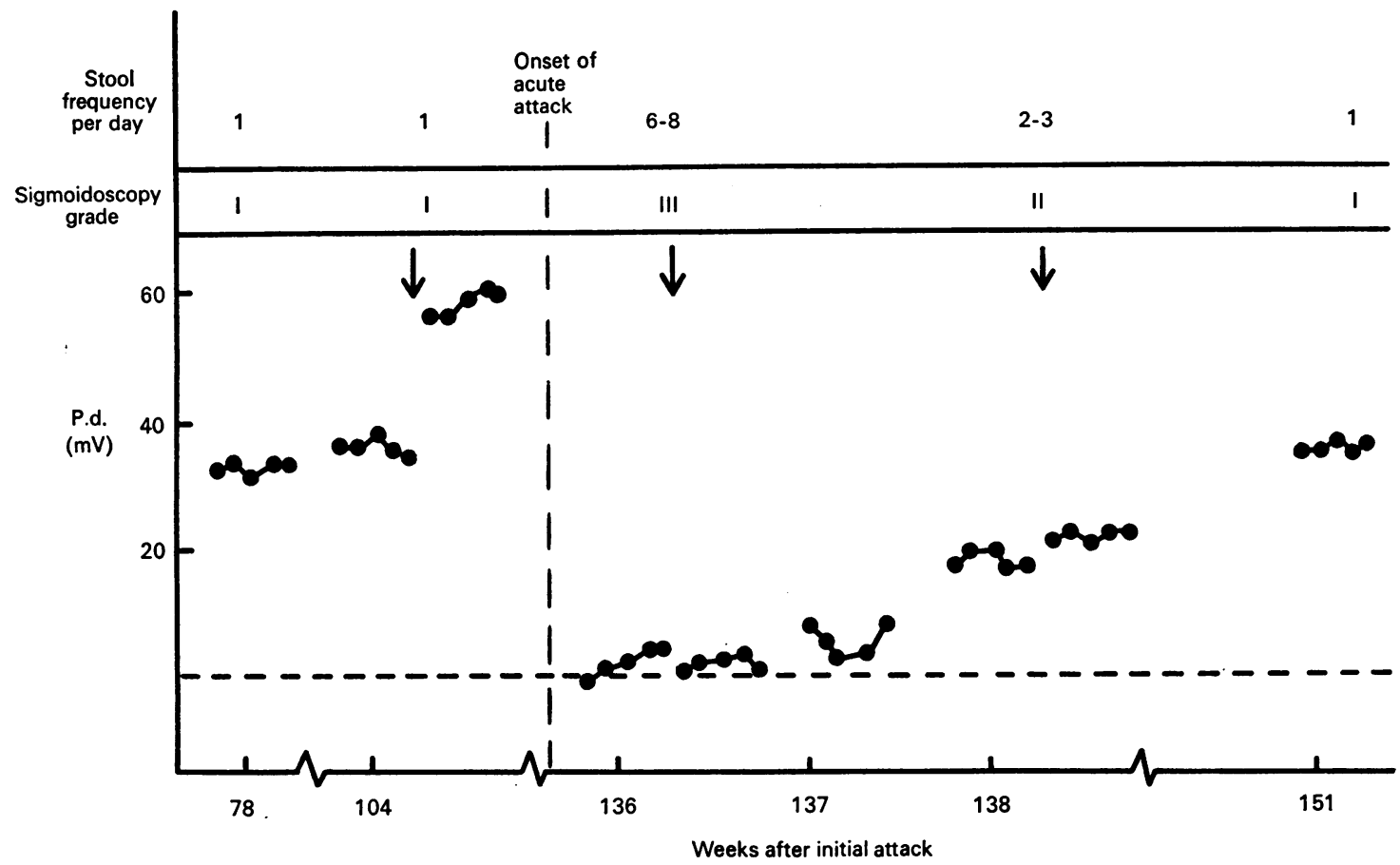

Fig 2 Transmucosal pd measurements from a longitudinal study on a man of 24 years during a recurrent episode of acute proctocolitis. Fludrocortisone, $0.5 \mathrm{mg}$, was given at the times marked with arrows to test the response to mineralocorticoid stimulation.

may nearly double within a few hours of commencing an aldosterone infusion. The change in pd is not, however, uniform along the length of the colon, being greatest in the distal colon and rectum (Edmonds and Marriott, 1967). These effectsare seen in man both with exogenous and endogenously produced aldosterone (Edmonds and Richards, 1970).

Carbenoxolone has a similar action and in some individuals the pd may be elevated as high as 60 to $80 \mathrm{mV}$ during several weeks of treatment, associated with increased sodium absorption and potassium secretion (Tomkins and Edmonds, 1975).

Spironolactone we have found tends to lower the pd in most individuals to less than $30 \mathrm{mV}$, but other diuretics in therapeutic doses seem to have little effect. Thyroid hormones also appear to be important, particularly when the epithelium is under stimulation by mineralocorticoids. The Na-K activated ATPases in the mucosa are substantially depleted in hypothyroidism, and, since these enzymes are probably of considerable importance in active sodium transport, their reduction may be the relevant factor (Thompson and Edmonds, 1974).

The functional state of the epithelium would also be expected to influence the potential difference. In acute proctocolitis, the pd is reduced practically to zero and stimulation with mineralocorticoids is without effect (fig 2). During recovery the pd gradually returns towards normal but the time taken for this to occur is variable and in some patients months or even years may elapse before the pd is restored (Edmonds, 1970). Associated with these changes in pd is considerable impairment of sodium absorption and increased permeability (Edmonds and Pilcher, 1973; Rask Madsen and Brix Jensen, 1973) and it seems likely that in colitis the lowered pd results both from cellular injury with damage to the sodium pump mechanism (reduction in E) and increased epithelial permeability (reduced $\mathbf{R}_{\mathbf{s}}$ ). Measurements of pd may well prove useful clinically as a guide to diagnosis and prognosis, as they offer a fairly simple objective means of assessing the state of the mucosa.

In conclusion, the last few years have seen the development of relatively simple techniques which allow the transmucosal pd of gut epithelia in man to be measured satisfactorily, providing there is access to the luminal side. Measurements made using a sigmoidoscope have allowed a number of studies to 
be carried out on the distal colon and rectum and some factors which influence the pd have been examined. The pd is of significance both because it considerably influences the flows of charged particles across the epithelium and also because it is a measurable variable which reflects something of the functional state of the tissue. Since, however, it is affected by a variety of factors, these must be defined and considered if any meaningful interpretation is to be made.

\section{References}

Andersson, S., and Grossman, M. I. (1965). Profile of pH, pressure and potential difference at astroduodenal junction in man. Gastroenterology, 49, 364-371.

Archampong, E. Q., and Edmonds, C. J. (1972). Effect of luminal ions on the transepithelial electrical potential difference of human rectum. Gut, 13, 559-565.

Archampong, E. Q., Harris, J., and Clark, C. G. (1972). The absorption and secretion of water and electrolytes across the healthy and the diseased human colonic mucosa measured in vitro. Gut, 13, 880-886.

Barry, R. J. C. (1967). Electrical changes in relation to transport. Brit. med. Bull., 23, 266-269.

Dalmark, M. (1970). The transmucosal electrical potential difference across human rectum in vivo following perfusion of different electrolyte solutions. Scand. J. Gastroent., 5, 421-426.

Diamond, J. M., and Harrison, S. C. (1966). The effect of membrane fixed charges on diffusion potentials and streaming potentials. J. Physiol. (Lond.), 183, 37-57.

Edmonds, C. J. (1970). Electrical potentials of the sigmoid colon and rectum in irritable bowel syndrome and ulcerative colitis. Gut, 11, 867-874.
Edmonds, C. J., and Marriott, J. (1967). The effect of aldosterone and adrenalectomy on the electrical potential difference of rat colon and on the transport of sodium, potassium, chloride and bicarbonate. J. Endocr., 39, 517-531.

Edmonds, C. J., and Marriott, J. C. (1968). Electrical potential and short circuit current of an in vitro preparation of rat colon mucosa. J. Physiol. (Lond.), 194, 479-494.

Edmonds, C. J., and Nielsen, O. E. (1968). Transmembrane electric potential differences and ionic composition of mucosal cells of rat colon. Acta physiol. scand., 72, 338-349.

Edmonds, C. J., and Pilcher, D. (1973). Electrical potential difference and sodium and potassium fluxes across rectal mucosa in ulcerative colitis. Gut, 14, 784-789.

Edmonds, C. J., and Richards, P. (1970). Measurement of rectal electrical potential difference as an instant screening test for hyperaldosteronism. Lancet, 2, 624-627.

Finkelstein, A., and Mauro, A. (1963). Equivalent circuits as related to ionic systems. Biophys. J., 3, 215-237.

Geall, M. G., Code, C. F., McIlrath, D. C., and Summerskill, W. H. J. (1969). Measurement of gastrointestinal transmural electric potential difference in man. Gut, 11, 34-37.

Grantham, R. N., Code, C. F., and Schlegel, J. F. (1970). Reference electrode sites in determination of potential difference across the gastrooesophageal mucosal junction. Mayo Clin. Proc., 45, 265-274.

Rask-Madsen, J., and Brix Jensen, P. (1973). Electrolyte transport capacity and electrical potentials of the normal and the inflamed human rectum in vivo. Scand. J. Gastroent., 8, 169-175.

Schultz, S. G. (1972). Electrical potential difference and electromotive forces in epithelial tissues. J. gen. Physiol., 59, 794-798.

Smyth, D. H., and Wright, E. M. (1966). Streaming potentials in the rat small intestine. J. Physiol. (Lond.), 182, 591-602.

Thompson, B. D., and Edmonds, C. J. (1974). Aldosterone, sodium depletion and hypothroidism on the ATPase activity of rat colonic epithelium. J. Endocr., 62, 489-496.

Tomkins, A., and Edmonds, C. J. (1975). Effect of carbenoxolone on the electrical potential difference and sodium and potassium transport across rectal mucosa. (In press).

\title{
The normal colonic bacterial flora
}

\author{
M. J. HILL AND B. S. DRASAR \\ From the Bacterial Metabolism Research Laboratory, Central Public Health Laboratory, \\ Colindale Hospital, London
}

Interest in the human intestinal bacterial flora has increased greatly in recent years. To a large extent this is because techniques have now been developed which permit the study of the dominant members of the gut flora, the non-sporing strictly anaerobic bacteria.

There are two major approaches to the study of the gut flora. The first is the classical bacteriological approach, the identification and the enumeration of the major groups of bacteria. The second is the study of the biochemical activities of the flora. These two aspects of the flora will be discussed in turn.

\section{The Normal Colonic Flora}

The normal colonic flora is usually inferred from the composition of the normal faecal flora, since suitable techniques for sampling various levels of the colon have yet to be developed. Data from animal studies would support the assumption that the flora does not alter during defaecation, indicating that the faecal flora adequately represent that of the rectosigmoid. We know that the flora of the recto-sigmoid differs from that at the ileocaecal junction (table I) and can infer, but no more, that the change takes 